DOI 10. 18307/2021. 0127

(c) 2021 by Journal of Lake Sciences

\title{
淡水磷酸盐氧同位素前处理方法的优化
}

\author{
薛 珂 ${ }^{1,2}$, 张润宇 $^{1 * *}$, 安 宁 $^{1}$, 陈敬安 ${ }^{1}$, 邹银洪 ${ }^{1}$, 刘 勇 $^{1,2}$, 张 志 $^{1,2}$ \\ (1: 中国科学院地球化学研究所,贵阳 550081) \\ (2: 中国科学院大学, 北京 100049)
}

\begin{abstract}
摘 要: 磷是地表水体中的关键性营养盐, 在水生生态系统的物质循环与能量流动方面发挥着重要作用, 研究水体中磷 的来源、转化与归趋对于了解水环境的演变过程与科学保护具有重要意义. 近年来, 磷酸盐氧同位素 $\left(\delta^{18} \mathrm{O}_{\mathrm{P}}\right)$ 技术已逐渐 应用于淡水环境中磷的来源示踪与生物地球化学循环研究, 其样品前处理主要沿用海水方法体系. 相比而言, 淡水样品 中 $\mathrm{PO}_{4}^{3-}$ 浓度通常较低, 有机质和干扰离子含量却较高, 复杂的样品前处理过程极大地制约了 $\delta^{18} \mathrm{O}_{\mathrm{P}}$ 分析在淡水环境体系 的广泛应用. 为此, 本研究针对现有海水样品 $\delta^{18} \mathrm{O}_{\mathrm{P}}$ 前处理方法在地表淡水环境的适用性加以检验, 并进行了三点优化改 进: (1) 将 MAGIC 沉淀步骤使用的 $\mathrm{MgCl}_{2}$ 替换为 $\mathrm{Mg}\left(\mathrm{NO}_{3}\right)_{2}$, 避免了 $\mathrm{Cl}^{-}$的干扰, 减少 $\mathrm{AgCl}$ 杂质的生成; (2)调节生成 $\mathrm{Ag}_{3} \mathrm{PO}_{4}$ 溶液 $\mathrm{pH}$ 值为 8.0, 保证 $\mathrm{Ag}_{3} \mathrm{PO}_{4}$ 沉淀快速完全; (3) 对 $\mathrm{Ag}_{3} \mathrm{PO}_{4}$ 沉淀过程采用避光处理, 降低了 $\mathrm{AgNO}_{3}$ 及 $\mathrm{Ag}_{3} \mathrm{PO}_{4}$ 可能的光解 影响, 提高了 $\mathrm{Ag}_{3} \mathrm{PO}_{4}$ 的纯度, 使 $\delta^{18} \mathrm{O}_{\mathrm{P}}$ 的测试结果更为准确. 本改进方法为后续利用 $\delta^{18} \mathrm{O}_{\mathrm{P}}$ 技术深人探究淡水环境中磷的 生物地球化学循环与生态环境效应提供了有益的方法借鉴.
\end{abstract}

关键词: 淡水环境; 磷; 磷酸盐氧同位素; 前处理方法; 改进

\section{Optimization approach of pretreatment methods for phosphate oxygen isotopic analysis in freshwater*}

\author{
Xue Ke ${ }^{1,2}$, Zhang Runyu ${ }^{1 * *}$, An Ning ${ }^{1}$, Chen Jing'an ${ }^{1}$, Zou Yinhong ${ }^{1}$, Liu Yong ${ }^{1,2}$ \& Zhang Zhi ${ }^{1,2}$ \\ (1: Institute of Geochemistry, Chinese Academy of Sciences, Guiyang 550081, P.R.China) \\ (2: University of Chinese Academy of Sciences, Beijing 100049, P.R.China)
}

Abstract: Phosphorus (P) is one of the key nutrients in surface water, and plays a vital role in the material cycle and energy flow in aquatic ecosystems. Hence, it is of great significance to explore the source, transformation, and fate in water body for understanding its evolution process and scientific protection of water environment. Recently, phosphate oxygen isotope technology $\left(\delta^{18} \mathrm{O}_{\mathrm{P}}\right)$ has been gradually applied in tracing the $\mathrm{P}$ source and studying the biogeochemical cycle in freshwater environment, which most used the sample pretreatment of seawater methods. By contrast, the concentrations of $\mathrm{PO}_{4}^{3-}$ in freshwater samples are $\mathrm{u}-$ sually low, whereas the contents of organic matter and interference ions are high. The pretreatment complexity restricted the extensive application of $\delta^{18} \mathrm{O}_{\mathrm{P}}$ analysis in freshwater ecosystems. This study examined the applicability of the existing pretreatment methods of seawater sample in surface freshwater environment, and further carried out three optimization improvements: (1)We replaced $\mathrm{MgCl}_{2}$ solution with $\mathrm{Mg}\left(\mathrm{NO}_{3}\right)_{2}$ solution in the MAGIC precipitation step to avoid the interference of $\mathrm{Cl}^{-}$and the impurities of AgCl. (2) The solution $\mathrm{pH}$ value of $\mathrm{Ag}_{3} \mathrm{PO}_{4}$ precipitation was adjusted to 8.0 to ensure the rapid and complete precipitation. (3) Possible photolysis effects of $\mathrm{AgNO}_{3}$ and $\mathrm{Ag}_{3} \mathrm{PO}_{4}$ were inhibited by using light avoidance. This modification improved the purity of $\mathrm{Ag}_{3} \mathrm{PO}_{4}$, and attained more accurate $\delta^{18} \mathrm{O}_{\mathrm{P}}$ results. The present method gives a valuable reference to further study of the biogeochemical cycle and eco-effects of $\mathrm{P}$ in freshwater environment using $\delta^{18} \mathrm{O}_{\mathrm{P}}$ analysis.

Keywords: Freshwater environment; phosphorus; phosphate oxygen isotope; pretreatment method; improvement

* 2020-04-19 收稿; 2020-05-28 收修改稿.

国家自然科学基金项目 (41573133,41273151) 和国家自然科学基金委员会-贵州喀斯特科学研究中心项目 ( U1612442) 联合资助.

** 通信作者;E-mail : zhangrunyu@ vip.gyig.ac.cn. 
磷是地表水环境中重要的营养元素之一, 其循环转化直接影响着水体水质、初级生产力水平及浮游植 物的种群演替 ${ }^{[1-2]}$. 在自然环境中, 磷仅有一个稳定同位素 $\left({ }^{31} \mathrm{P}\right)$, 无法用以示踪其来源与循环过程 ${ }^{[3]}$. 幸运 的是, 磷通常以磷酸盐的形式存在, 而磷酸盐中磷与氧紧密结合, P-O 键能为 $359.8 \mathrm{~kJ} / \mathrm{mol}$, 在地表自然温度 和 $\mathrm{pH}$ 等无机环境下几乎不会水解发生氧原子交换, 由此可以借助磷酸盐氧同位素 $\left(\delta^{18} \mathrm{O}_{\mathrm{P}}\right)$ 组成进行磷的来 源示踪 ${ }^{[4]}$. 在一定的生物或酶作用下, 磷酸盐还可与周围水体发生氧同位素交换, 进而使 $\delta^{18} \mathrm{O}_{\mathrm{P}}$ 趋于同位素 平衡分馏值,基于此也能推断磷的生物地球化学循环过程 ${ }^{[5-6]}$.

随着 $\delta^{18} \mathrm{O}_{\mathrm{P}}$ 测试技术与同位素比值质谱仪 (IRMS) 的快速发展, 近年来已在海洋、河流、湖泊、湿地等地 表水体中磷的迁移转化研究领域得到了广泛的应用 ${ }^{[7-15]}$. 目前对水体 $\delta^{18} \mathrm{O}_{\mathrm{P}}$ 的前处理方法主要是从海水体系 中演变而来, 一种为先用氢氧化钠和氯化镁反应生成絮状氢氧化镁沉淀, 吸附并富集水中的可溶性磷酸盐 (SRP), 该方法称为镁共沉淀法 (MAGIC), 再用硝酸溶解后加人铈盐生成磷酸铈沉淀进行纯化, 再经阳离子 交换树脂去除 $\mathrm{Ce}^{3+[16]}$; 另一种通过磷钼酸铵和磷酸铵镁沉淀纯化 $\mathrm{PO}_{4}^{3-}$, 再用阴阳离子交换树脂去除金属离 子和有机质 ${ }^{[17]}$, 二者最后均转化成磷酸银 $\left(\mathrm{Ag}_{3} \mathrm{PO}_{4}\right)$ 进行 $\delta^{18} \mathrm{O}_{\mathrm{P}}$ 的测定. 总体来看, 这些方法通常操作过程繁 琐, 耗时较长, 生成 $\mathrm{Ag}_{3} \mathrm{PO}_{4}$ 受 $\mathrm{pH}$ 影响较大 ${ }^{[18-19]}$. 淡水环境样品中的 $\mathrm{PO}_{4}^{3-}$ 浓度一般较低, 而有机质和 $\mathrm{Cl}^{-}$、 $\mathrm{Ca}^{2+} 、 \mathrm{Mg}^{2+}$ 等含量较高, 非常容易干扰 $\mathrm{Ag}_{3} \mathrm{PO}_{4}$ 的生成和 $\delta^{18} \mathrm{O}_{\mathrm{P}}$ 的准确分析 ${ }^{[20-22]}$. 因此有必要对现有 $\delta^{18} \mathrm{O}_{\mathrm{P}}$ 分析 前处理方法在地表淡水环境样品中的适用性加以检验, 并进行相应地改进研究, 以期建立一种更为高效、准 确, 且适宜于淡水样品 $\delta^{18} \mathrm{O}_{\mathrm{P}}$ 分析的前处理方法.

\section{1 实验部分}

\section{1 试剂与材料}

试剂: 磷酸二氢钾 $\left(\mathrm{KH}_{2} \mathrm{PO}_{4}\right)$ 、六水硝酸镁 $\left(\mathrm{Mg}\left(\mathrm{NO}_{3}\right)_{2} \cdot 6 \mathrm{H}_{2} \mathrm{O}\right)$ 、氯化镁 $\left(\mathrm{MgCl}_{2}\right)$ 、氢氧化钠 $(\mathrm{NaOH}) 、 乙$ 酸钾 $\left(\mathrm{CH}_{3} \mathrm{COOK}\right)$ 、浓硝酸 $\left(\mathrm{HNO}_{3}\right)$ 、醋酸 $\left(\mathrm{CH}_{3} \mathrm{COOH}\right)$ 、浓氨水 $\left(\mathrm{NH}_{3} \cdot \mathrm{H}_{2} \mathrm{O}\right) 、 30 \%$ 双氧水 $\left(\mathrm{H}_{2} \mathrm{O}_{2}\right)$ 从国药集团 化学试剂有限公司购买, 均为分析纯以上; 硝酸银 $\left(\mathrm{AgNO}_{3}, 99.9 \%\right)$ 购买自 Alfa Aesar 公司, 硝酸铈 $\left(\mathrm{Ce}\left(\mathrm{NO}_{3}\right)_{3} \cdot 6 \mathrm{H}_{2} \mathrm{O}, 99.5 \%\right)$ 从 Acros Organics 公司获得.

材料:50 mL 普通离心管、50 mL 黑色不透光离心管 (Argos Technologies)、250 ml 聚乙烯瓶、阳离子交换 树脂( Biorad AG 50W-X8, $\mathrm{H}^{+}$型, 100-200 目).

标准试剂:包括购买自 Alfa Aesar 公司的 $\mathrm{Ag}_{3} \mathrm{PO}_{4}$ (简称 $\mathrm{alfa}, 99.9 \%, \mathrm{GR}$ ), 两种实验室内部 $\mathrm{Ag}_{3} \mathrm{PO}_{4}$ 标准 $\mathrm{KL}\left(\delta^{18} \mathrm{O}_{\mathrm{P}}=11.3 \% 0\right)$ 和 $\mathrm{KH}\left(\delta^{18} \mathrm{O}_{\mathrm{P}}=20.0 \%\right.$ ), 3 种国际原子能机构 ( International Atomic Energy Agency, IAEA) 的含氧标准物质 $\mathrm{NBS} 127\left(\mathrm{BaSO}_{4}, \delta^{18} \mathrm{O}=9.3 \% 0\right) 、 601$ ( Benzoic Acid, $\left.\delta^{18} \mathrm{O}=23.3 \% 0\right)$ 和 $\mathrm{USG}^{-32}\left(\mathrm{KNO}_{3}, \delta^{18} \mathrm{O}=\right.$ $25.7 \%$ ) .

模拟实验溶液配制: 根据我国地表水湖库 II 类、 $\mathrm{V}$ 类以及污水一级 $\mathrm{A}$ 标准的总磷 ( TP ) 浓度國值 ${ }^{[23-24]}$, 设置 3 种梯度系列磷溶液, 浓度分别为 $0.02 、 0.2$ 和 $0.5 \mathrm{mg} / \mathrm{L}$, 使用 $\mathrm{KH}_{2} \mathrm{PO}_{4}$ 和超纯水进行配制. 其中, 浓度为 $0.02 \mathrm{mg} / \mathrm{L}$ 的样品配制 $50 \mathrm{~L}$, 浓度为 0.2 和 $0.5 \mathrm{mg} / \mathrm{L}$ 的溶液各配制 $20 \mathrm{~L}$.

污水样品:采自贵阳市某污水处理厂两份平行样品,标记为 $1^{\#}-1$ 和 $1^{\#}-2$. 采集 $20 \mathrm{~L}$, 现场立即用 $0.45 \mu \mathrm{m}$ 醋酸纤维滤膜过滤后, 冷藏运回实验室进行 $\delta^{18} \mathrm{O}_{\mathrm{P}}$ 分析前处理实验. 经分析, 水样 $\mathrm{TP}$ 浓度为 $1.3 \mathrm{mg} / \mathrm{L}$, 溶解 态反应磷 $(\mathrm{SRP})$ 为 $0.8 \mathrm{mg} / \mathrm{L}$, 溶解性有机碳 $(\mathrm{DOC})$ 浓度为 $50 \pm 7 \mathrm{mg} / \mathrm{L}$.

\section{2 实验方法}

$\delta^{18} \mathrm{O}_{\mathrm{P}}$ 分析的前处理参照 McLaughlin 方法 ${ }^{[16]}$, 主要包括 3 个步骤: (1)添加 $\mathrm{NaOH}$ 和 $\mathrm{MgCl}_{2}$, 通过 MAGIC 共沉淀法富集溶液中的 $\mathrm{SRP}$; 2) 用 $\mathrm{HNO}_{3}$ 溶解上述 $\mathrm{Mg}(\mathrm{OH})_{2}$ 沉淀, 后加人 $\mathrm{Ce}\left(\mathrm{NO}_{3}\right)_{3}$ 生成 $\mathrm{CePO}_{4}$ 沉淀, 再次富 集磷, 通过 Biorad AG 50W-X8 树脂除去 $\mathrm{Ce}^{3+}$; 3加人 $\mathrm{AgNO}_{3}$ 溶液生成 $\mathrm{Ag}_{3} \mathrm{PO}_{4}$ 沉淀, 并用 $\mathrm{H}_{2} \mathrm{O}_{2}$ 去除有机质的 干扰.

在此基础上, 我们验证了该方法在上述不同浓度模拟磷溶液以及污水样品的 $\delta^{18} \mathrm{O}_{\mathrm{P}}$ 测定情况, 并针对主 要的不足之处, 进行了如下改进 (图 1) : (1) 将 MAGIC 步骤使用的 $\mathrm{MgCl}_{2}$ 替换为 $\mathrm{Mg}\left(\mathrm{NO}_{3}\right)_{2}$, 减少 $\mathrm{Cl}^{-}$的干扰, 从而降低减少氯化银 $(\mathrm{AgCl})$ 杂质的生成. (2)对比不同 $\mathrm{pH}$ 值条件下 $\mathrm{Ag}_{3} \mathrm{PO}_{4}$ 的生成情况, 探讨 $\mathrm{Ag}_{3} \mathrm{PO}_{4}$ 生成过 
程的最佳 $\mathrm{pH}$ 值. (3)进行室内暗光实验, 并使用黑色不透光离心管, 降低 $\mathrm{AgNO}_{3}$ 及生成 $\mathrm{Ag}_{3} \mathrm{PO}_{4}$ 可能的遇光分 解效应, 尽量提高样品的纯度, 确保 $\delta^{18} \mathrm{O}_{\mathrm{P}}$ 的测试准确.

对于较大体积的含磷模拟溶液与污水样品, 按照图 1 的 $\delta^{18} \mathrm{O}_{\mathrm{P}}$ 前处理流程, 由于每个样品在 MAGIC 步 骤生成的 $\mathrm{Mg}(\mathrm{OH})_{2}$ 沉淀过多, 视情况分成了 2 4 组同时进行后续实验.

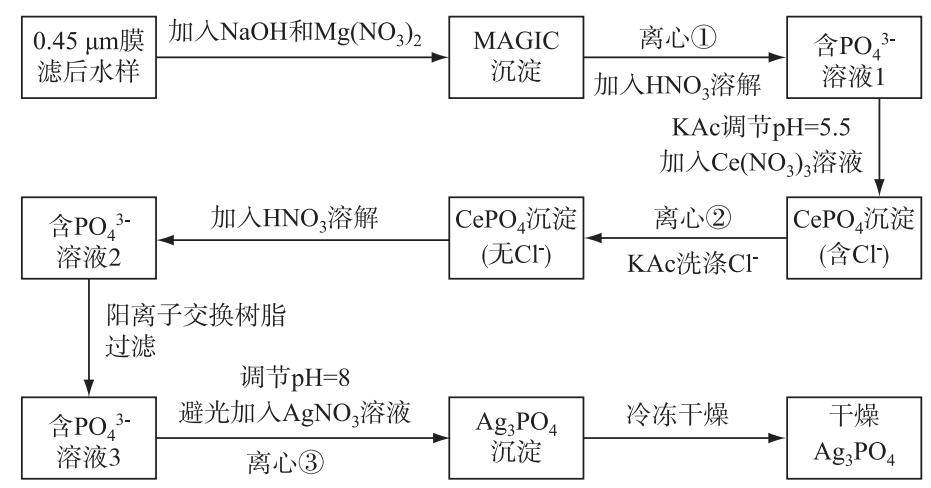

图 1 改进的淡水样品 $\delta^{18} \mathrm{O}_{\mathrm{P}}$ 前处理流程示意图 (1)(2)(3)处测定磷的回收率)

Fig.1 Schematic diagram of the improved pretreatment methods for $\delta^{18} \mathrm{O}_{\mathrm{P}}$ analysis in freshwater samples

(The phosphorus recovery rate was determined in (1)(2)(3) steps, respectively)

\section{3 样品分析与表征}

水样及溶液磷浓度的测定: 高磷浓度的样品采用钼锑抗分光光度法测量 (适用浓度范围为 $0.01 \sim 0.6$ $\mathrm{mg} / \mathrm{L}$ ), 低磷浓度的样品采用磷锄杂多酸一孔雀绿分光光度法测量 ( 适用浓度范围 $0.001 \sim 0.3 \mathrm{mg} / \mathrm{L}$ ) ${ }^{[24]}$.

$\delta^{18} \mathrm{O}_{\mathrm{P}}$ 分析: 称取 $0.45 \sim 0.50 \mathrm{mg}$ 的 $\mathrm{Ag}_{3} \mathrm{PO}_{4}$ 样品包入银舟, 高温裂解后, 在氦气载气下, $\mathrm{Ag}_{3} \mathrm{PO}_{4}$ 裂解释放 的 $\mathrm{O}_{2}$ 与石墨发生反应生成 $\mathrm{CO}, \mathrm{CO}$ 通过连续流系统载人 IRMS 测量 $\delta^{18} \mathrm{O}_{\mathrm{P}}$ 值, 同时每隔 5 个样品插人氧同位 素标准物质, 以判断数据的稳定性. $\delta^{18} \mathrm{O}_{\mathrm{P}}$ 值均是相对于 VSMOW.

为验证 $\mathrm{Ag}_{3} \mathrm{PO}_{4}$ 样品的纯度, 使用 $\mathrm{X}$ 射线衍射技术 ( $\mathrm{XRD}$, Empyream, 荷兰), 样品质量不少于 $200 \mathrm{mg}$, 使 用玛瑙研针磨细过 325 目篮, 制成长 $\times$ 宽在 $3 \mathrm{~cm} \times 3 \mathrm{~cm}$ 以内、上下表面至少一面平整、厚度不超过 $3 \mathrm{~mm}$ 的薄 片,上机对生成的 $\mathrm{Ag}_{3} \mathrm{PO}_{4}$ 样品进行图谱分析,以获得其成分、结构、形态等信息.

为测定 $\mathrm{Ag}_{3} \mathrm{PO}_{4}$ 样品中有机质的含量, 称取约 $300 \mathrm{mg}$ 的 $\mathrm{Ag}_{3} \mathrm{PO}_{4}$ 样品包人锡舟, 进人燃烧管在纯氧氛围 下静态充分燃烧, 燃烧的最后阶段再通人定量的动态氧气以保证所有的有机物和无机物都完全燃烧, 使用 有机元素分析仪 (vario Macro cube, 德国) 对样品进行元素分析, 以获得其 $\mathrm{C} 、 \mathrm{H} 、 \mathrm{O} 、 \mathrm{~N}$ 等元素的含量.

\section{2 结果与讨论}

\section{$2.1 \delta^{18} \mathrm{O}_{\mathrm{P}}$ 测试方法的准确度与精密度}

通过 3 种 IAEA 含氧标准物质 NBS127、601 和 USG-32 的多点校准 (图 2), 测量的 $\mathrm{KH} \delta^{18} \mathrm{O}_{\mathrm{P}}$ 值为 $20.0 \% 0 \pm 0.2 \%, \mathrm{KL}$ $\delta^{18} \mathrm{O}_{\mathrm{P}}$ 值为 $11.3 \%{ }_{0} \pm 0.2 \%$, 与内部标准值相一致. Alfa 磷酸银 的 $\delta^{18} \mathrm{O}_{\mathrm{P}}$ 值为 $20.4 \%{ }_{0} \pm 0.4 \%$. 另外, 从多次标准物质平行分析 的结果来看, $\mathrm{NBS} 127$ 的 $\delta^{18} \mathrm{O}$ 值为 $9.3 \% \pm 0.2 \% 0,601$ 的 $\delta^{18} \mathrm{O}$ 值为 $23.3 \% \pm 0.2 \%$, USG -32 的 $\delta^{18} \mathrm{O}$ 值为 $25.7 \% \pm 0.2 \%$, 实验 过程中分析误差均小于 $0.5 \%$, 精密度高,进一步证明了本实 验室仪器对于测试 $\delta^{18} \mathrm{O}_{\mathrm{P}}$ 的可靠性. 从图 2 也可以发现, KL

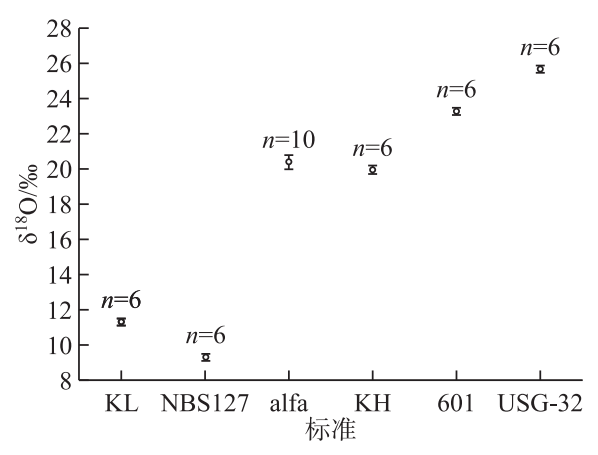

图 2 不同标准样品的 $\delta^{18} \mathrm{O}$ 测量值 和 NBS127 的 $\delta^{18} \mathrm{O}$ 值较为偏负, alfa 和 $\mathrm{KH}$ 的 $\delta^{18} \mathrm{O}$ 值很接 Fig. $2 \delta^{18} \mathrm{O}$ values of different standard samples 近,601 和 USG 的 $\delta^{18} \mathrm{O}$ 值更偏正. 
此外, 本文还总结了目前已发表的淡水环境样品中不同环境介质中 $\delta^{18} \mathrm{O}_{\mathrm{P}}$ 组成特征 ${ }^{[4-15]}$. 河流 $\delta^{18} \mathrm{O}_{\mathrm{P}}$ 为 $8.6 \% 0 \sim 17.2 \%$, 湖泊 $\delta^{18} \mathrm{O}_{\mathrm{P}}$ 为 $8.4 \% 0 \sim 17.1 \%$, 地下水 $\delta^{18} \mathrm{O}_{\mathrm{P}}$ 为 $9.0 \% 0 \sim 15.1 \%$, 污水 $\delta^{18} \mathrm{O}_{\mathrm{P}}$ 为 $8.4 \% 0 \sim 18.4 \%$, 洗涤 剂 $\delta^{18} \mathrm{O}_{\mathrm{P}}$ 为 $13.3 \% \sim 18.6 \%$, 化粪池 $\delta^{18} \mathrm{O}_{\mathrm{P}}$ 为 $13.2 \% 0 \sim 14.5 \%$. 通常, 不同水体 $\delta^{18} \mathrm{O}_{\mathrm{P}}$ 的变化范围有所不同, 主要 与磷的来源组成以及生物活动程度等密切相关 ${ }^{[4,15]}$. 相对而言, 地下水 $\delta^{18} \mathrm{O}_{\mathrm{P}}$ 相对偏负, 而湖泊、河流等受人 类活动的影响, $\delta^{18} \mathrm{O}_{\mathrm{P}}$ 偏正. 污水、化粪池中受洗涤剂等含磷物质输人所致, 微生物活动也较为剧烈, $\delta^{18} \mathrm{O}_{\mathrm{P}}$ 更 趋于平衡值.

自然界淡水样品 $\delta^{18} \mathrm{O}_{\mathrm{P}}$ 值大多介于 $8 \%$ 20\% 之间, 可以使用 $\mathrm{KL} / \mathrm{NBS} 127 、 \mathrm{alfa}^{-} \mathrm{Ag}_{3} \mathrm{PO}_{4} / \mathrm{KH}$ 及 $601 / \mathrm{USG}-$ 323 对标准物质组合进行 $\delta^{18} \mathrm{O}_{\mathrm{P}}$ 的分析校正, 也可以简化使用 NBS127、601 和 USG-32 进行校准( 图 2), 这 3 种标准目前已多用于环境样品的 $\delta^{34} \mathrm{~S} 、 \delta^{13} \mathrm{C} 、 \delta^{15} \mathrm{~N}$ 分析.

\section{2 模拟淡水样品的分析}

2.2.1 磷的回收率 我们对比了不同磷浓度和不同处理方法的样品各步骤回收率的差异 (表 1). 结果表明, 在 MAGIC 和 $\mathrm{CePO}_{4}$ 两步骤磷的回收率较高, 而离子交换树脂步骤较低, 导致最终生成 $\mathrm{Ag}_{3} \mathrm{PO}_{4}$ 的磷回收率为 $57.0 \% \sim 86.4 \%$, 这是因为在树脂分离过程中一部分磷滞留在洗脱柱上. 随着含磷模拟溶液磷浓度的升高, 磷 的回收率显著提高. 对于高磷浓度的样品, 丢失的部分占比较小, 对整体回收率的影响不大; 但对低浓度的 样品, 丢失部分占比较大, 对整体回收率的影响也较大. 因此, 对于低浓度磷酸盐的淡水样品, 需采集大体积 的样品进行多次 MAGIC 预富集, 并重复进行 Biorad AG 50W-X8 树脂柱洗脱处理, 以确保磷的回收率 ${ }^{[17-18]}$. 值得注意的是,使用 $\mathrm{Mg}\left(\mathrm{NO}_{3}\right)_{2}$ 进行 MAGIC 沉淀与原先的 $\mathrm{MgCl}_{2}$ 处理对磷回收率的影响并不大.

表 $1 \delta^{18} \mathrm{O}_{\mathrm{P}}$ 前处理各步骤中磷的回收率

Tab. 1 The phosphorus recovery rate in each step of the $\delta^{18} \mathrm{O}_{\mathrm{P}}$ pretreatment methods

\begin{tabular}{|c|c|c|c|c|c|c|}
\hline 名称 & $\begin{array}{c}\text { 初始 SRP 浓度/ } \\
(\mathrm{mg} / \mathrm{L})\end{array}$ & $\begin{array}{c}\text { MAGIC 步骤 } \\
\text { 所用试剂 }\end{array}$ & $\begin{array}{c}\text { MAGIC 步骤 } \\
\text { 回收率/\% }\end{array}$ & $\begin{array}{c}\mathrm{CePO}_{4} \text { 步骤 } \\
\text { 回收率/\% }\end{array}$ & $\begin{array}{c}\text { 离子交换树脂 } \\
\text { 回收率/\% }\end{array}$ & $\begin{array}{c}\mathrm{Ag}_{3} \mathrm{PO}_{4} \text { 步骤 } \\
\text { 回收率/\% }\end{array}$ \\
\hline $\mathrm{A} 1$ & 0.02 & $\mathrm{MgCl}_{2}$ & 99.3 & 94.1 & 61.7 & 57.6 \\
\hline $\mathrm{A} 2$ & 0.02 & $\mathrm{Mg}\left(\mathrm{NO}_{3}\right)_{2}$ & 99.5 & 93.3 & 61.4 & 57.0 \\
\hline B1 & 0.2 & $\mathrm{MgCl}_{2}$ & 98.8 & 94.7 & 73.2 & 68.5 \\
\hline B2 & 0.2 & $\mathrm{Mg}\left(\mathrm{NO}_{3}\right)_{2}$ & 97.3 & 97.0 & 68.4 & 64.6 \\
\hline $\mathrm{C} 1$ & 0.5 & $\mathrm{MgCl}_{2}$ & 99.2 & 98.8 & 72.8 & 71.4 \\
\hline $\mathrm{C} 2$ & 0.5 & $\mathrm{Mg}\left(\mathrm{NO}_{3}\right)_{2}$ & 99.8 & 97.3 & 68.3 & 76.3 \\
\hline $1^{\#}-1$ & 0.8 & $\mathrm{Mg}\left(\mathrm{NO}_{3}\right)_{2}$ & 98.7 & 99.9 & 87.6 & 86.4 \\
\hline $1^{\#}-2$ & 0.8 & $\mathrm{Mg}\left(\mathrm{NO}_{3}\right)_{2}$ & 99.1 & 99.7 & 82.4 & 84.3 \\
\hline
\end{tabular}

2.2.2 模拟淡水样品的 $\delta^{18} \mathrm{O}_{\mathrm{P}}$ 值 我们使用 $\mathrm{KH}_{2} \mathrm{PO}_{4}$ 纯水配制的 3 种磷浓度为 $0.02 、 0.2 、 0.5 \mathrm{mg} / \mathrm{L}$ 的溶液进行 $\delta^{18} \mathrm{O}_{\mathrm{P}}$ 前处理实验, 对比使用 $\mathrm{MgCl}_{2}$ 和 $\mathrm{Mg}\left(\mathrm{NO}_{3}\right)_{2}$ 进行 MAGIC 沉淀. 由于 $\mathrm{A} 2$ 实验组中原溶液磷浓度过低, 因 此生成的 $\mathrm{Ag}_{3} \mathrm{PO}_{4}$ 沉淀较少, 无法进行多次测量. 对于其他含磷模拟溶液经不同前处理生成的 $\mathrm{Ag}_{3} \mathrm{PO}_{4}$ 样品, 均在 IRMS 上进行 3 次以上的 $\delta^{18} \mathrm{O}_{\mathrm{P}}$ 测试.

由图 3 可见, 采用 $\mathrm{MgCl}_{2}$ 进行 MAGIC 沉淀的 3 个不同浓度系列的磷溶液最后生成的 $\mathrm{Ag}_{3} \mathrm{PO}_{4}$ 样品 $\delta^{18} \mathrm{O}_{\mathrm{P}}$ 平均值为 $12.28 \%$, 明显偏离 $\mathrm{KH}_{2} \mathrm{PO}_{4}$ 和 $\mathrm{AgNO}_{3}$ 溶液直接合成的 $\mathrm{Ag}_{3} \mathrm{PO}_{4}$ 的 $\delta^{18} \mathrm{O}_{\mathrm{P}}$ 值 $(13.1 \%)$, 尤其是 $\mathrm{C} 1$ 组标 准偏差高达 $0.7 \%$. 这可能由于 $\mathrm{Cl}^{-}$未能彻底去除, 最后生成的 $\mathrm{Ag}_{3} \mathrm{PO}_{4}$ 含有 $\mathrm{AgCl}$, 进而导致数据测量的偏差. 目前去除 $\mathrm{Cl}^{-}$的方法为使用缓冲溶液 (使用 $\mathrm{KAc}$ 和 $\mathrm{HAc}$ 将 $\mathrm{pH}$ 调解为 5.6 的超纯水) 洗涤 3 遍 ${ }^{[21]}$, 我们建议 多洗涤几遍,并用 $\mathrm{AgNO}_{3}$ 溶液鉴定是否仍有残留 $\mathrm{Cl}^{-}$.

使用 $\mathrm{Mg}\left(\mathrm{NO}_{3}\right)_{2}$ 替代后, $\mathrm{Ag}_{3} \mathrm{PO}_{4}$ 样品 $\delta^{18} \mathrm{O}_{\mathrm{P}}$ 的平均值为 $13.18 \%$, 更接近于用 $\mathrm{KH}_{2} \mathrm{PO}_{4}$ 和 $\mathrm{AgNO}_{3}$ 溶液直接 合成的 $\mathrm{Ag}_{3} \mathrm{PO}_{4}$ 的 $\delta^{18} \mathrm{O}_{\mathrm{p}}$ 值, 并且最大标准偏差为 $0.46 \%$ ( $\mathrm{C} 2$ 组), 在该仪器分析方法可接受的范围之内 $\left(<0.5 \%\right.$ ) . 实验证明, 使用 $\mathrm{Mg}\left(\mathrm{NO}_{3}\right)_{2}$ 替代 $\mathrm{MgCl}_{2}$ 进行 $\mathrm{MAGIC}$ 沉淀后, 可有效减少 $\mathrm{Cl}^{-}$的干扰, 大大提高 $\mathrm{Ag}_{3} \mathrm{PO}_{4}$ 的纯度, 使 $\delta^{18} \mathrm{O}_{\mathrm{P}}$ 测定结果更加接近真实值. 


\section{3 生成 $\mathrm{Ag}_{3} \mathrm{PO}_{4}$ 样品的 XRD 分析}

由 2.2 节可见,传统方法使用 $\mathrm{MgCl}_{2}$ 进行 MAGIC 沉淀, 可能会导致 $\mathrm{Cl}^{-}$的不完全去除, 最后生成 $\mathrm{AgCl}$ 杂质, 使得样 品不纯. 同时, 研究还表明, 如果不进行严格的避光操作, $\mathrm{AgNO}_{3}$ 和 $\mathrm{Ag}_{3} \mathrm{PO}_{4}$ 光解也会产生 $\mathrm{Ag}$ 单质, 同样也会影响最终 样品的纯度及 $\delta^{18} \mathrm{O}_{\mathrm{P}}$ 值 ${ }^{[21]}$. 按照 Blake 法 ${ }^{[25]}$, 加人银氨溶液 后加热使氨水挥发析出 $\mathrm{Ag}_{3} \mathrm{PO}_{4}$, 亮黄色的结晶浮于溶液表 面. 而在 McLaughlin 法 ${ }^{[16]}$, 调节 $\mathrm{pH}$ 至 7.0 8.0, 加人 $\mathrm{AgNO}_{3}$ 溶液, 迅速生成黄色的 $\mathrm{Ag}_{3} \mathrm{PO}_{4}$ 沉淀, 但沉淀随时间慢慢变 暗. 我们于是对 $\mathrm{Mg}\left(\mathrm{NO}_{3}\right)_{2}$ 替代实验后 $\mathrm{Ag}_{3} \mathrm{PO}_{4}$ 的生成实验 分为室内正常光照和地下室暗光及使用黑色不透光离心管 盛放 $\mathrm{Ag}_{3} \mathrm{PO}_{4}$ 加以比较研究, 并对改进前后的 $\mathrm{Ag}_{3} \mathrm{PO}_{4}$ 样品进 行 XRD 检验, 判断是否产生上述 $\mathrm{Ag}_{3} \mathrm{PO}_{4}$ 的光解现象.

从图 4 可以看出, 与直接合成 $\mathrm{Ag}_{3} \mathrm{PO}_{4}$ 相比, 使用 $\mathrm{MgCl}_{2}$ 进行 MAGIC 沉淀最终的样品中, 由于 $\mathrm{Cl}^{-}$的未完全去除, 的 确含有 $\mathrm{AgCl}$ 杂质. 在 $\mathrm{Mg}\left(\mathrm{NO}_{3}\right)_{2}$ 实验组未避光样品中确实 检测到 $\mathrm{Ag}$ 单质的存在, 而严格避光处理后样品较为纯净, 没有发现 $A g$ 单质. 前人研究表明, 造成 $A g$ 单质的生成主要 有两点原因:一是加人 $\mathrm{AgNO}_{3}$ 溶液生成 $\mathrm{Ag}_{3} \mathrm{PO}_{4}$ 沉淀后, 一 少部分 $\mathrm{AgNO}_{3}$ 附着在 $\mathrm{Ag}_{3} \mathrm{PO}_{4}$ 表面, 在 $\mathrm{NH}_{3} \cdot \mathrm{H}_{2} \mathrm{O}$ 还原作用下 转变为 $\mathrm{Ag}$ 单质; 二是 $\mathrm{Ag}_{3} \mathrm{PO}_{4}$ 在有水和光照条件下也会在 表面分解产生灰黑色的 $\mathrm{Ag}$ 单质 ${ }^{[16-17]}$. $\mathrm{Ag}$ 单质的存在会导 致上机时氧元素信号偏低,增大测试的数据误差 ${ }^{[21]}$.

为此, 有人使用氨水重结晶和缓慢微沉淀法有效减少 $\mathrm{Ag}$ 单质 ${ }^{[26-27]}$ 的产生, 可用 $10 \mathrm{~mL}$ 浓氨水溶解 $\mathrm{Ag}_{3} \mathrm{PO}_{4}$, 通过 $0.2 \mu \mathrm{m}$ 聚碳酸酯膜收集溶液, 在过滤过程中,灰黑色杂质被去除, 因为它们不会溶解在氨水中. 过滤的溶液收集在 $50 \mathrm{~mL}$ 离心管中, $40^{\circ} \mathrm{C}$ 下烘干,进一步发现氨水蒸发后重新沉淀出亮黄色 $\mathrm{Ag}_{3} \mathrm{PO}_{4} . \mathrm{Xu}$ 等 ${ }^{[27]}$ 进一步指出要尽量缩短氨水重结晶的时间, 最好在沉淀后半小时内完成. 尽管高浓度 $\mathrm{NH}_{4} \mathrm{NO}_{3}$ 重复冲 洗可以降低 $\mathrm{Ag}$ 单质的影响, 但通常建议避免这种措施, 因为随着 $\mathrm{pH}$ 值的变化, 不溶性 $\mathrm{AgO}$ 可能会形成, 进 而导致 $\mathrm{Ag}_{3} \mathrm{PO}_{4}$ 的污染. 况且前人方法操作繁琐、耗时较长, 且在生成 $\mathrm{Ag}_{3} \mathrm{PO}_{4}$ 后进行操作, 会对样品造成一定 的损失. 相比之下, 本文采用暗光实验结合避光处理, 直接避免了 $\mathrm{Ag}$ 单质的生成且更加经济快捷. 此外, 为 了防止 $\mathrm{AgNO}_{3}$ 与 $\mathrm{Ag}_{3} \mathrm{PO}_{4}$ 的光解, 应尽量控制 $\mathrm{AgNO}_{3}$ 的投加量, 不要太过量, 混合溶液中 $\mathrm{Ag}: \mathrm{P}$ 浓度比例不宜 超过 $13^{[26]}$.

\section{4 污水样品分析}

2.4.1 $\mathrm{pH}$ 值对 $\mathrm{Ag}_{3} \mathrm{PO}_{4}$ 生成的影响研究 在 $\delta^{18} \mathrm{O}_{\mathrm{P}}$ 分析的前处理中, $\mathrm{pH}$ 值条件对于 $\mathrm{Ag}_{3} \mathrm{PO}_{4}$ 生成至关重要, 常 用的实验方法中 $\mathrm{Ag}_{3} \mathrm{PO}_{4}$ 生成的 $\mathrm{pH}$ 条件一般为 7.0 8. $0^{[16,27]}$. Mine 等的研究表明 $\mathrm{Ag}_{3} \mathrm{PO}_{4}$ 沉淀的适合 $\mathrm{pH}$ 范 围较宽, 为 $7.0 \sim 10.0^{[26]}$. 但是, 究竟 $\mathrm{Ag}_{3} \mathrm{PO}_{4}$ 生成的最佳 $\mathrm{pH}$ 值条件是多少目前仍然不清楚. 因此, 我们采用 污水样品, 设置了 3 组 $\mathrm{pH}$ 值, 分别为 $\mathrm{pH}=7.0 、 8.0$ 和 9.0, 每组 $\mathrm{pH}$ 值分别设置 3 组平行实验, 对比不同 $\mathrm{pH}$ 条 件下生成 $\mathrm{Ag}_{3} \mathrm{PO}_{4}$ 的情况.

我们发现 $\mathrm{pH}=9.0$ 时几乎没有生成 $\mathrm{Ag}_{3} \mathrm{PO}_{4}$ 沉淀, $\mathrm{pH}$ 值为 7.0 和 8.0 时均可快速生成 $\mathrm{Ag}_{3} \mathrm{PO}_{4}$ 沉淀, 但 $\mathrm{pH}=8.0$ 时生成量为 $\mathrm{pH}=7.0$ 时的 $1.5 \sim 3$ 倍. 同时, 从 $\delta^{18} \mathrm{O}_{\mathrm{p}}$ 结果来看 (图 5), 样品 $1^{\#}-1$ 在 $\mathrm{pH}=7.0$ 和 8.0 时, $\delta^{18} \mathrm{O}_{\mathrm{P}}$ 平均值分别为 $15.9 \%$ 和 $15.7 \%$, 样品 $1^{\#}-2$ 对应 $15.2 \%$ 和 $15.6 \%$, 所有数据最大标准偏差为 $0.44 \%$, 可以 看出这两个 $\mathrm{pH}$ 条件下对 $\delta^{18} \mathrm{O}_{\mathrm{P}}$ 值的差别影响不大.

研究表明, 当溶液 $\mathrm{pH}$ 低于 7.0 时, $\mathrm{Ag}_{3} \mathrm{PO}_{4}$ 难以完全沉淀, 且已生成的沉淀也易在酸性条件下溶解 ${ }^{[26]}$. 

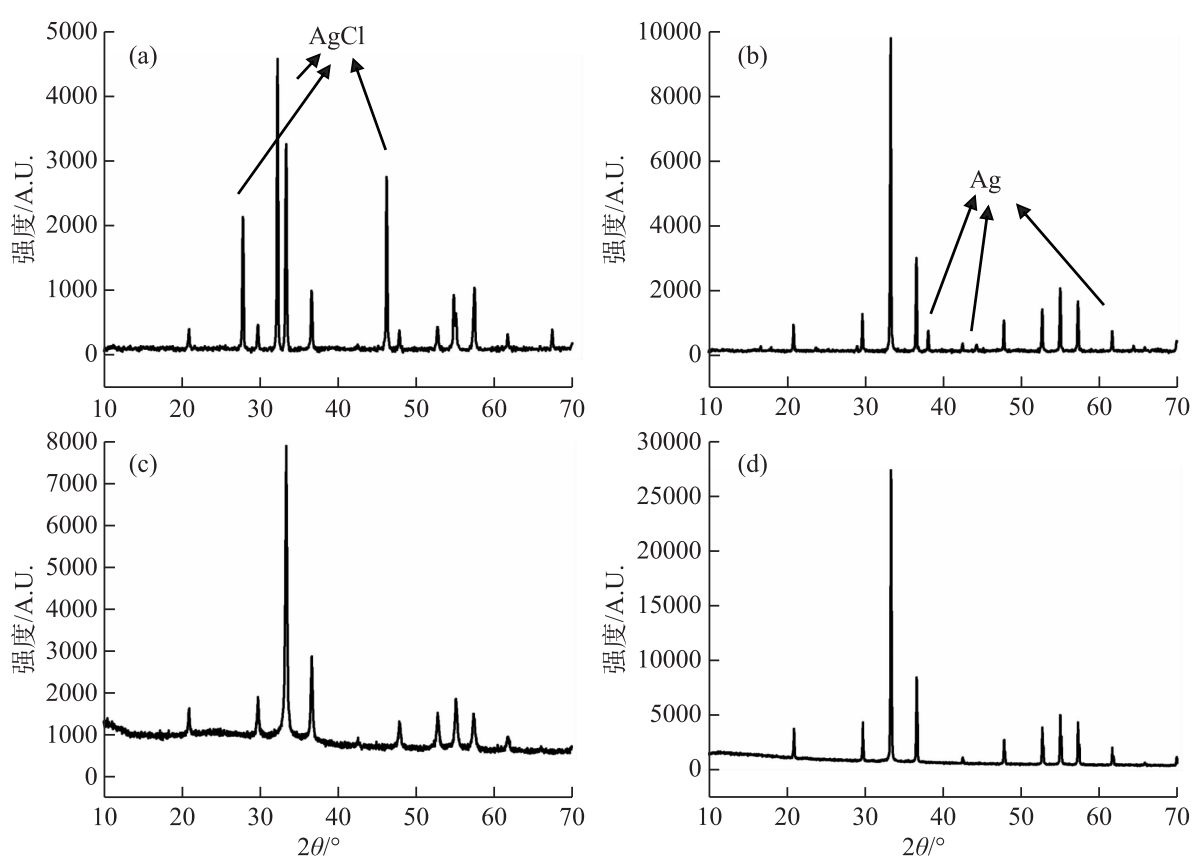

图 4 前处理方法改进前后样品的 XRD 图谱

( $\mathrm{a}$ : 使用 $\mathrm{MgCl}_{2}$ 进行 MAGIC 沉淀且未完全去除 $\mathrm{Cl}^{-}$; b : 使用 $\mathrm{Mg}\left(\mathrm{NO}_{3}\right)_{2}$ 进行 MAGIC 沉淀,

但未完全避光; $\mathrm{c}$ : 使用 $\mathrm{Mg}\left(\mathrm{NO}_{3}\right)_{2}$ 进行 MAGIC 沉淀且避光处理; $\mathrm{d}$ : 直接合成 $\left.\mathrm{Ag}_{3} \mathrm{PO}_{4}\right)$

Fig. 4 XRD patterns of samples before and after the improvement methods

(a: $\mathrm{MgCl}_{2}$ was used for MAGIC precipitation and $\mathrm{Cl}^{-}$was not completely removed;

b: MAGIC precipitation was performed with $\mathrm{Mg}\left(\mathrm{NO}_{3}\right)_{2}$, but not completely avoided light;

c: MAGIC precipitation with $\mathrm{Mg}\left(\mathrm{NO}_{3}\right)_{2}$ using the dark treatment; d: direct synthesis of $\left.\mathrm{Ag}_{3} \mathrm{PO}_{4}\right)$

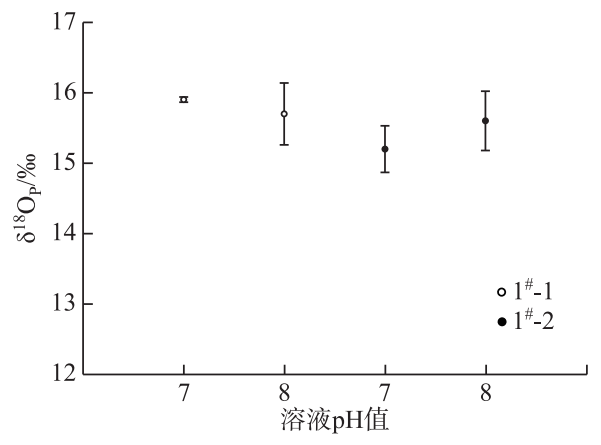

图 5 溶液 $\mathrm{pH}=7.0 、 8.0$ 时不同样品的 $\delta^{18} \mathrm{O}_{\mathrm{P}}$ 值

Fig. $5 \delta^{18} \mathrm{O}_{\mathrm{P}}$ values of different samples at solutions $\mathrm{pH}=7.0$ and 8.0

当 $\mathrm{pH}$ 值过高时, 特别是 $\mathrm{pH}$ 大于 9.0 时, 可能生成 $\mathrm{AgOH}$ 沉淀, 最后转化为 $\mathrm{Ag}_{2} \mathrm{O}$, 进而影响 $\delta^{18} \mathrm{O}_{\mathrm{P}}$ 值的准确性 ${ }^{[28]}$. 为此, 我们建议 $\mathrm{pH}=8.0$ 为 $\mathrm{Ag}_{3} \mathrm{PO}_{4}$ 生成的最佳条件, 这与 Dettman 等 ${ }^{[29]}$ 提出的快速微沉淀法条件非常吻合.

2.4.2 有机质的去除 为了验证 $\mathrm{Ag}_{3} \mathrm{PO}_{4}$ 中有机质的去除 程度, 我们分析了 4 个样品中 $\mathrm{C} 、 \mathrm{~N}$ 的元素含量 (表 2). 其 中, Alfa 公司购买的 $\mathrm{Ag}_{3} \mathrm{PO}_{4}$ 的 $\mathrm{C} 、 \mathrm{~N}$ 含量最低, 直接合成的 $\mathrm{Ag}_{3} \mathrm{PO}_{4}$ 的 $\mathrm{C} 、 \mathrm{~N}$ 含量也较低,分别为 $0.564 \%$ 和 $0.078 \% . \mathrm{C} 1$ 为使用 $\mathrm{MgCl}_{2}$ 对模拟溶液 $\mathrm{C} 1$ 进行 MAGIC 沉淀生成的 $\mathrm{Ag}_{3} \mathrm{PO}_{4}$, 而样品 $1^{\#}$ 为使用 $\mathrm{Mg}\left(\mathrm{NO}_{3}\right)_{2}$ 进行 MAGIC 沉淀生 成的 $\mathrm{Ag}_{3} \mathrm{PO}_{4}$, 二者含 $\mathrm{C}$ 量接近, 与直接合成的 $\mathrm{Ag}_{3} \mathrm{PO}_{4}$ 相 当. 经计算, 样品 $1^{\#}$ 的 DOC 去除率达到 $90 \%$ 以上. 此外,

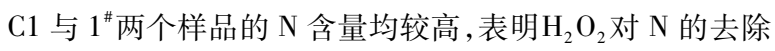
效果不够理想. Grimes 等 ${ }^{[30]}$ 和张秀梅等 ${ }^{[22]}$ 也发现该方法 对有机质的去除效率没有 Colman 的方法 ${ }^{[17]}$ 高, 因为后者特别增加了针对有机质除去阴离子交换树脂的步 骤. 为此, 对于一些有机质含量较高的工业废水与生活污水样品, 建议参照卢阳阳等 ${ }^{[19]}$ 改进的 McLaughlin 法, 在除去 $\mathrm{Ce}^{3+}$ 后, 采用阴离子交换树脂去除有机质的干扰. Xu 等 ${ }^{[27]}$ 使用 XAD-2 树脂去除有机物, 制备的 
$\mathrm{Ag}_{3} \mathrm{PO}_{4}$ 中 $\mathrm{C} 、 \mathrm{~N}$ 含量分别为 $0.226 \% \pm 0.033 \%$ 和 $0.030 \% \pm$ $0.006 \%$, 对有机物的去除率较高. Liu 等 ${ }^{[21]}$ 向湖泊沉积物样 品提取液中加人无磷活性炭粉振荡 $30 \mathrm{~min}$ 后过滤去除有机 质, 发现该方法效果良好, 而且可以避免磷的损失, 也是一个 适用的简便选择.

\section{3 结论}

本研究针对现有淡水样品 $\delta^{18} \mathrm{O}_{\mathrm{P}}$ 前处理方法的局限性,
表 $2 \mathrm{Ag}_{3} \mathrm{PO}_{4}$ 样品中 $\mathrm{C} 、 \mathrm{~N}$ 含量

Tab.2 C and $\mathrm{N}$ contents in $\mathrm{Ag}_{3} \mathrm{PO}_{4}$ samples

\begin{tabular}{ccc}
\hline 样品 & $\mathrm{C} / \%$ & $\mathrm{~N} / \%$ \\
\hline $\mathrm{Alfa}_{\mathrm{Ag}} \mathrm{PO}_{4}$ & 0.018 & 0.014 \\
直接合成 $\mathrm{Ag}_{3} \mathrm{PO}_{4}$ & 0.564 & 0.078 \\
$\mathrm{C} 1$ & 0.454 & 0.941 \\
$1^{\#}$ & 0.634 & 0.379 \\
\hline
\end{tabular}
采用 $\mathrm{Mg}\left(\mathrm{NO}_{3}\right)_{2}$ 代替 $\mathrm{MgCl}_{2}$ 进行 MAGIC 沉淀,有效降低 $\mathrm{AgCl}$ 的生成,特别是严格的避光操作可避免 $\mathrm{Ag}_{3} \mathrm{PO}_{4}$ 光解生成 $\mathrm{Ag}$ 单质, 提高样品的纯度. 同时, 优化调节 $\mathrm{Ag}_{3} \mathrm{PO}_{4}$ 生成溶液 $\mathrm{pH}$ 值为 8.0 ,使沉淀过程快速完全进行, 确保了样品 $\delta^{18} \mathrm{O}_{\mathrm{P}}$ 分析结果的可靠性.

研究结果证明,改进的 $\delta^{18} \mathrm{O}_{\mathrm{P}}$ 前处理方法解决了目前淡水样品 $\delta^{18} \mathrm{O}_{\mathrm{P}}$ 前处理过程存在的操作步骤繁琐、产 生 $\mathrm{AgCl}$ 杂质干扰及 $\mathrm{Ag}_{3} \mathrm{PO}_{4}$ 光解等不良影响, 既简化了磷酸盐的富集、纯化过程, 又保证了 $\delta^{18} \mathrm{O}_{\mathrm{P}}$ 分析的准确 性, 是一种适宜于淡水样品 $\delta^{18} \mathrm{O}_{\mathrm{P}}$ 前处理方法, 有望极大地推动淡水环境中磷的来源示踪与生态环境效应研究.

\section{4 参考文献}

[ 1 ] Schelske CL. Eutrophication: Focus on phosphorus. Science, 2009, 324: 722.

[ 2 ] Huser BJ, Futter M, Lee JT et al. In-lake measures for phosphorus control: The most feasible and cost-effective solution for long-term management of water quality in urban lakes. Water Research, 2016, 97: 142-152. DOI: 10.1016/j.watres.2015. 07.036 .

[ 3 ] Paytan A, McLaughlin K. Tracing the sources and biogeochemical cycling of phosphorus in aquatic systems using isotopes of oxygen in phosphate//Handbook of Environmental Isotope Geochemistry. Berlin Heidelberg: Springer, 2011. DOI: 10. 1007/978-3-642-10637-8_21.

[ 4 ] Davies CL, Surridge BWJ, Gooddy DC. Phosphate oxygen isotopes within aquatic ecosystems: Global data synthesis and future research priorities. Science of the Total Environment, 2014, 496: 563-575. DOI: 10.1016/j.scitotenv.2014.07.057.

[ 5 ] Liang Y, Blake RE. Oxygen isotope studies of biogeochemical cycling of phosphorus. Organic Geochemistry, 2006, 37: $1263-1277$.

[ 6 ] O’Neil JR, Vennemann TW, McKenzie WF. Effects of speciation on equilibrium fractionations and rates of oxygen isotope exchange between $\left(\mathrm{PO}_{4}\right)$ aq and $\mathrm{H}_{2}$ O. Geochimica et Cosmochimica Acta, 2003, 67 (17): 3135-3144. DOI: 10.1016/ S0016-7037 ( 02$)$ 00970-5.

[ 7 ] Elsbury KE, Paytan A, Ostrom NE et al. Using oxygen isotopes of phosphate to trace phosphorus sources and cycling in Lake Erie. Environmental Science \& Technology, 2009, 43(9) : 3108-3114. DOI : 10.1021/es8034126.

[ 8 ] Jaisi DP, Blake RE. Tracing sources and cycling of phosphorus in Peru Margin sediments using oxygen isotopes in authigenic and detrital phosphates. Geochimica et Cosmochimica Acta, 2010, 74(11) : 3199-3212. DOI: 10.1016/j.gca.2010. 02.030 .

[ 9 ] McLaughlin K, Kendall C, Silva SR et al. Phosphate oxygen isotope ratios as a tracer for sources and cycling of phosphate in North San Francisco Bay, California. Journal of Geophysical Research: Biogeosciences, 2006, 111(3): G03003. DOI: 10.1029/2005JG000079.

[10] Pistocchi C, Tamburini F, Gruau G et al. Tracing the sources and cycling of phosphorus in river sediments using oxygen isotopes: Methodological adaptations and first results from a case study in France. Water Research, 2017,111 : $346-356$. DOI: $10.1016 /$ j.watres.2016.12.038.

[11] Granger SJ, Heaton THE, Pfahler V et al. The oxygen isotopic composition of phosphate in river water and its potential sources in the Upper River Taw catchment, UK. Science of the Total Environment, 2017, 574: 680-690. DOI: 10.1016/j. scitotenv.2016.09.007.

[12] Tonderski K, Andersson L, Lindström G et al. Assessing the use of $\delta^{18} \mathrm{O}$ in phosphate as a tracer for catchment phosphorus sources. Science of the Total Environment, 2017, 607/608: 1-10. DOI: 10.1016/j.scitotenv.2017.06.167. 
[13] Yuan HZ, Li Q, Kukkadapu RK et al. Identifying sources and cycling of phosphorus in the sediment of a shallow freshwater lake in China using phosphate oxygen isotopes. Science of the Total Environment, 2019, 676: 823-833. DOI : 10.1016/ j. scitotenv.2019.04.322.

[14] Lin JJ, Chen ZG, Liu GS. The preconcentration, separation and purification of phosphate for the seawater dissolved phosphate oxygen isotope composition analysis. Journal of Xiamen University: Natural Science, 2010, 49(2) : 238-241. [林继 军, 陈志刚, 刘广山. 应用于磷酸盐氧同位素测定的海水中溶解态磷酸盐的富集、分离与纯化. 厦门大学学报: 自 然科学版, 2010, 49(2): 238-241.]

[15] Tian LY, Guo QJ, He HJ et al. Progress on phosphate oxygen isotope technology in environmental science. Chinese Journal of Ecology, 2016, 35(7) : 1947-1956. [田丽艳, 郭庆军, 何会军等. 磷酸盐氧同位素技术在环境科学中的研究进 展. 生态学杂志, 2016, 35(7) : 1947-1956.]

[16] McLaughlin K, Silva S, Kendall C et al. A precise method for the analysis of $\delta^{18} \mathrm{O}$ of dissolved inorganic phosphate in seawater. Limnology and Oceanography: Methods, 2004, 2(7) : 202-212. DOI: 10.4319/lom.2004.2.202.

[17] Colman AS. The oxygen isotope composition of dissolved inorganic phosphate and the marine phosphorus cycle [Dissertation]. Connecticut: Yale University, 2002.

[18] Cid-Andres AP. A review on useful concepts for stable isotope of oxygen in phosphate $\left(\delta^{18} \mathrm{O}_{\mathrm{P}}\right)$ extraction, purification and analysis of freshwater samples and other potential phosphate sources. Microchemical Journal, 2015, 123: 105-110. DOI: 10.1016/j.microc.2015.05.021.

[19] Lu YY, Zheng ZZ, Yin XJ et al. The measurement of oxygen isotope composition of dissolved inorganic phosphate in seawater. Acta Geoscientica Sinica, 2012, 33(6) : 961-966. [卢阳阳, 郑珍珍, 尹希杰等. 海水溶解磷酸盐氧同位素组 成的测定. 地球学报, 2012, 33(6): 961-966.]

[20] Tcaci M, Barbecot F, Helie J et al. A new technique to determine the phosphate oxygen isotope composition of freshwater samples at low ambient phosphate concentration. Environmental Science and Technology, 2019, 53(17) : 10288-10294. DOI: $10.1021 /$ acs.est.9b00631.

[21] Liu Y, Wang JF, Chen JG et al. Pretreatment method for the analysis of phosphate oxygen isotope $\left(\delta^{18} \mathrm{O}_{\mathrm{P}}\right)$ of different phosphorus fractions in freshwater sediments. Science of the Total Environment, 2019, 685: 229-238. DOI: 10.1016/j.scitotenv.2019.05.238.

[22] Zhang XM, Wang YR, Ma SZ et al. Comparison of pretreatment methods of oxygen isotope composition of phosphate in lake sediments. J Lake Sci, 2017, 29(2) : 512-520. DOI: 10.18307/2017.0227. [张秀梅, 王亚惢, 马书占等. 湖泊沉 积物中磷酸盐氧同位素前处理方法对比. 湖泊科学, 2017, 29(2) : 512-520.]

[23] Discharge standard of pollutants for municipal wastewater treatment plants. Beijing: Ministry of Ecology and Environment of the People's Republic of China, 2016. GB/T 18918-2016. [城镇污水处理厂污染物排放标准. 北京: 中华人民共和 国生态环境部, 2016. GB/T 18918-2016.]

[24] Editorial Board of “Water and wastewater monitoring and analysis methods”, Ministry of Environmental Protection of the People's Republic of China ed. Water and wastewater monitoring and analysis methods: 4th edition. Beijing: China Environmental Science Press, 2002. [ 国家环境保护总局《水和废水监测分析方法》编委会. 水和废水监测分析方法: 第 4 版. 北京: 中国环境科学出版社, 2002.]

[25] Blake RE, Chang SJ, Lepland A. Phosphate oxygen isotopic evidence for a temperate and biologically active Archaean ocean. Nature, 2010, 464(7291) : 1029-1032. DOI: 10.1038/nature08952.

[26] Mine AH, Waldeck A, Olack G et al. Microprecipitation and $\delta^{18} \mathrm{O}$ analysis of phosphate for paleoclimate and biogeochemistry research. Chemical Geology, 2017, 460: 1-14. DOI: 10.1016/j.chemgeo.2017.03.032.

[27] Xu ZF, Huang T, Yin XJ. Improvements in the preparation of phosphate for oxygen isotope analysis from soils and sediments. PLoS One, 2018, 13(9) : e0204203. DOI: 10.1371/journal.pone.0204203.

[28] Biedermann G, Sillén LG, Lindberg B et al. Studies on the hydrolysis of metal ions. part 30. A critical survey of the solubility equilibria of $\mathrm{Ag}_{2} \mathrm{O}$. Acta Chemica Scandinavica, 1960, 14: 717-725. DOI: 10.3891/acta.chem.scand.14-0717.

[29] Dettman DL, Kohn MJ, Quade J et al. Seasonal stable isotope evidence for a strong Asian monsoon throughout the past 10. 7 m.y. Geology, 2001, 29(1) : 31. DOI: 10.1130/0091-7613(2001)0290031 : ssiefa>2.0.co;2.

[30] Grimes V, Pellegrini M. A comparison of pretreatment methods for the analysis of phosphate oxygen isotope ratios in bioapatite. Rapid Communications in Mass Spectrometry, 2013, 27(3) : 375-390. DOI: 10.1002/rcm.6463. 